\title{
Sacrococcygeal teratoma in the perinatal period
}

\author{
R Tuladhar, S K Patole, J S Whitehall
}

Teratomas are formed when germ cell tumours arise from the embryonal compartment. The name is derived from the Greek word "teratos" which literally means "monster". The ending “-oma” denotes a neoplasm. ${ }^{1}$

\section{Incidence}

Sacrococcygeal teratoma is the most common congenital tumour in the neonate, reported in approximately $1 / 35000$ to $1 / 40000$ live births. ${ }^{2}$ Approximately $80 \%$ of affected infants are female-a $4: 1$ female to male preponderance. $^{2}$

The first reported case was inscribed on a Chaldean cuneiform tablet dated approximately $2000 \mathrm{BC}^{3}$ In the modern era, the first large series of infants and children with sacrococcygeal teratomas was reported by Gross et al in $1951 .{ }^{4}$

\section{Embryonic origin}

Sacrococcygeal teratomas have tissues derived from ectoderm, mesoderm, and endoderm. ${ }^{2}$ Although their embryonic origin is still uncertain, they are believed to arise early in gestation (at around the late second or early third week) from the totipotential cells of Hensen's node (also called the primitive knot), a remnant of the primitive streak in the coccygeal region..$^{5-7}$ The primitive streak appears as a linear thickening in the ectoderm at the caudal edge of the bilaminar embryonic disc. It usually diminishes in size, eventually disappearing after undergoing degenerative changes. As the mesoderm rapidly proliferates, the primitive streak comes to lie more and more caudally, where the remnant of Hensen's node descends to the tip or anterior surface of coccyx..$^{5-7}$

\section{Types}

Sacrococcygeal teratomas may be classified as benign (mature) and malignant or immature (composed of embryonic elements). ${ }^{8}$ Mature teratomas are most common in neonates $(68 \%)$ and older children (73\%). Immature teratomas are cystic, whereas malignant tumours are solid. Over $50 \%$ of sacrococcygeal teratomas have calcification and ossification. ${ }^{8}$ Keslar et al reported that $69(62 \%)$ of the 96 sacrococcygeal teratomas in their series were composed of both solid and cystic elements. ${ }^{8}$ The cysts may be filled with serous fluid, mucoid, or sebaceous material and lined by true epithelium. Ein et al found cystic tumours that were filled with cerebrospinal fluid from choroid plexus present in the tumour mass. ${ }^{6}$ Virtually any tissue can be present in a sacrococcygeal teratoma. ${ }^{810-13}$ Neuroglial tissue, skin, respiratory and enteric epithelium, cartilage, smooth muscel, and striated muscle are the most common elements found. ${ }^{8}$ Bone, pancreatic tissue, choroid plexus, and adrenal tissues are less commonly identified. ${ }^{12}$ An ocular lens present as lentinoids (lens-like cells), as well as a completely formed eye, have been found within sacrococcygeal teratomas. ${ }^{10}{ }^{11}$ Parizek et al reported a mature teratoma containing the lower half of a human body in one of fraternal twins. ${ }^{13}$

\section{Size}

Size of a sacrococcygeal teratoma (average 8 $\mathrm{cm}$, range 1 to $30 \mathrm{~cm}$ ) does not predict its biological behaviour. ${ }^{8}$ Altman et al have defined the size of sacrococcygeal teratomas as follows: small, 2 to $5 \mathrm{~cm}$ diameter; moderate, 5 to 10 $\mathrm{cm}$ diameter; large, $>10 \mathrm{~cm}$ diameter. ${ }^{14}$

\section{Site}

The sacrococcygeal region is the most common location. Less common sites are the mediastinum, testes, retroperitoneum, brain, head and neck, vagina, stomach, and pineal region. ${ }^{815}$ Sacrococcygeal teratomas may continue to grow posteriorly to form an external protrusion, or dissect anteriorly, distorting regional organs (rectum, vagina, and bladder) without invading them. ${ }^{8}{ }^{14}$

\section{Extent}

The American Academy of Pediatrics' surgical section (APPSS) classification helps in grading the extent of sacrococcygeal teratomas, as follows ${ }^{16}$ :

Type I-Predominantly external with minimal presacral component.

Type II-Present externally but with significant intrapelvic extension.

Type III-Apparent externally but predominantly a pelvic mass extending into the abdomen.

Type IV-Presacral with no external presentation.

\section{Histology}

Sacrococcygeal teratomas are graded histologically as follows ${ }^{17}$ :

Grade 0-Tumour contains only mature tissue.

Grade 1-Tumour contains rare foci of immature tissues.

Grade 2-Tumour contains moderate quantities of immature tissues.

Grade 3-Tumour contains large quantities of immature tissue with or without malignant yolk sac elements.

Grading of sacrococcygeal teratomas, unlike that of ovarian teratomas, does not seem to correlate directly with prognosis. ${ }^{8}$

\section{Fetal diagnosis}

Though described as early as the 19th century, it was not until the advent of antenatal ultrasonography that sacrococcygeal teratomas 


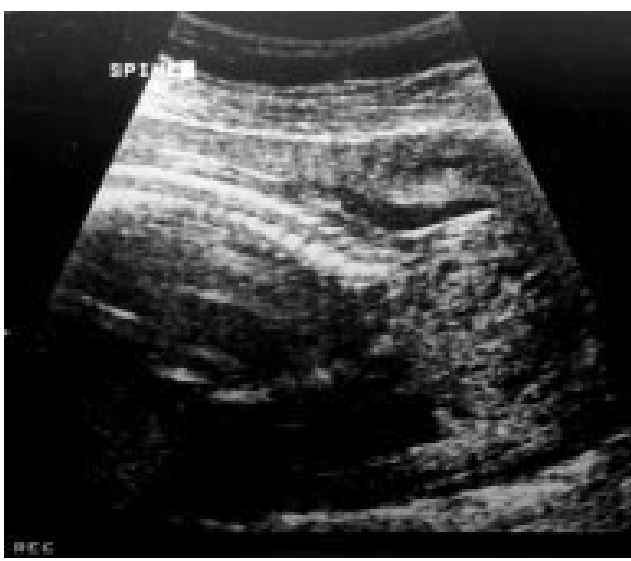

Figure 1 Antenatal ultrasound (saggital section) at 22 weeks of gestation, revealing a large sacrococcygeal teratoma (courtesy of Sinh Le and the Department of Radiology, Kirwan Hospital for Women).

were diagnosed in utero ${ }^{18}$ (fig 1 ). The majority of sacrococcygeal teratomas present between the $22 \mathrm{nd}$ and the 34 th week of gestation. Most are benign, resectable after birth without significant morbidity, and rarely associated with other congenital anomalies. There is thus the potential to lead a normal life without disability. ${ }^{18}$ The diagnosis of sacrococcygeal teratoma on routine sonograms is associated with a greater than expected incidence of prenatal and perinatal complications. ${ }^{19}$ Close antenatal follow up is needed to optimise patient counselling and treatment in the presence of a completely solid tumour and the onset of polyhydramnios. A poor outcome is usually correlated with placentomegaly, cardiomegaly, or non-immune hydrops fetalis. ${ }^{19}$

\section{Associated malformations}

The incidence of various congenital malformations associated with sacrococcygeal teratoma ranges from $5 \%$ to $26 \% .^{46}{ }^{20}$ Of these, anorectal and genital malformations are of prime concern. The association of sacrococcygeal teratoma with anorectal malformations was described as early as $1935 .{ }^{21}$ During the third week of embryonic life, genital folds unite to form the genital tubercle by migration of mesenchymal cells from the primitive streak region around the cloacal membrane. During the fourth and seventh weeks, the cloaca is subdivided by the urorectal septum to form the anorectal canal and the primitive urogenital sinus. ${ }^{21}$ A growing sacrococcygeal teratoma could thus encroach between the layers of the cloacal membrane and prevent descent and fusion of the urorectal septum to the cloacal membrane, resulting in a high anorectal malformation with a rectourethral fistula. ${ }^{21}$ An anterior location of the tumour could thus result in the absence of rectum and anus. ${ }^{21}$ The physical presence of a teratoma could also prevent fusion of the genital folds, resulting in a bifid scrotum and hypospadias. ${ }^{21}$

Other associated anomalies include spinal dysraphism, sacral agenesis, dislocation of the hips caused by a large tumour, and meningocele. ${ }^{8}$ Rarely cardiac anomalies such as a ventricular septal defect or gastrointestinal anomalies other than imperforate anus have been described. ${ }^{6}{ }^{14}$ Vogl and Riel reported a case with anorectal malformation, sacral dysplasia, and a presacral mass (Currarino's triad). ${ }^{22}$ Lahdenne et al reported vertebral abnormalities in $80 \%$ of their 45 patients with benign sacrococcygeal teratoma. ${ }^{23}$

\section{Perinatal management}

The perinatal management of sacrococcygeal teratoma requires an interdisciplinary liaison between obstetricians, neonatologists, and paediatric surgeons. The high perinatal/neonatal mortality and morbidity rates associated with sacrococcygeal teratoma are attributed to preterm delivery and to complications such as malignant invasion, haemorrhage into the tumour, obstruction of umbilical flow, high output cardiac failure, hydrops fetalis, and bladder outlet obstruction. ${ }^{19}{ }^{24-28}$ Some of these complications can be detected prenatally and treated appropriately. ${ }^{29}$ Garcia et al reported a case of decompression of a cystic grade IV sacrococcygeal teratoma in utero through an amniotic catheter to alleviate hydronephrosis and potential renal damage. ${ }^{30}$ Bond et al postulated that the tumour acts as an arteriovenous fistula, resulting in high output cardiac failure with placentomegaly and non-immune hydrops. If this were true then intervention to control this circulatory steal may be the only way to salvage severely affected fetuses. ${ }^{26}$ Close follow up with repeated ultrasound scans is necessary to monitor an increase in tumour size, extension into adjacent structures, tumour vascularity, and evidence of cardiac failure. ${ }^{18}$ Volume reduction amniocentesis and tocolysis may be required to treat symptomatic polyhydramnios and prevent preterm delivery. Barring the development of hydrops or maternal complications, delivery should be delayed to allow for fetal maturation. If hydrops develops, close maternal-fetal observation is needed. Maternal corticosteroids are advisable to accelerate fetal pulmonary maturation. ${ }^{31}$ Termination of pregnancy may have to be considered in cases with bad prognostic signs (for example, cardiac failure, placentomegaly, marked hydrops) or with associated major congenital anomalies. Kum et al described the following prerequisites for consideration of fetal intervention in preterm pregnancy ${ }^{18}$ :

- accurate prenatal diagnosis and well defined natural history to allow the confident diagnosis of a correctable lesion that will otherwise prevent fetal survival;

- absence of other life threatening or debilitating anomalies;

- ability to perform the procedure without increased risk to the mother's life or her future fertility.

The combination of fetal hydrops and sacrococcygeal teratoma is considered to be lethal. ${ }^{32}$ Nakayama et al, however, reported two such cases who survived after preterm delivery by caesarean section. ${ }^{32}$ They found that the combination of sacrococcygeal teratoma and hydrops had a particular effect on pulmonary function and fluid balance. Removal of tumour led to an immediate improvement in respira- 
tory function. They proposed the following tumour related factors contributing to respiratory insufficiency:

- the increased blood volume represented by tumour circulation may have exceeded the capacity of baby's lungs to arterialise blood;

- venous blood from the tumour may have contributed to a low mixed venous $\mathrm{PO}_{2}$ and pulmonary vasoconstriction; excision may have removed the source of desaturated acidaemic blood and relieved pulmonary vasoconstriction;

- fetal ascites and abdominal distension may have embarrassed lung development in utero, resulting in reduced lung volumes;

- ascites raised the diaphragm after birth and paracentesis thus improved pulmonary function.

\section{Investigations}

Sacrococcygeal teratomas should be excised soon after delivery to avoid tumour ulceration and haemorrhage and to reduce the risk of malignant change. ${ }^{18}$ The following investigations are recommended before surgery:

- $x$ rays of spine, abdomen, chest, and pelvis to detect evidence of tumour or metasta$\operatorname{sis}^{33}$;

- ultrasound to define the extent, nature (cystic or solid) of the mass, the presence and size of solid elements or calcification, and to recognise intrapelvic extension ${ }^{35}$;

- computed tomography to delineate the bony pelvic structures and to identify small areas of intrapelvic extension, but this involves radiation of the gonads; it is best performed following administration of a contrast agent ${ }^{835}$;

- magnetic resonance imaging (MRI) also delineates bony and muscular pelvic structures and can characterise the nature of the mass and detect intrapelvic extension ${ }^{8}{ }^{36}$; Hata et al reported facilitation of antenatal diagnosis of sacrococcygeal teratoma by combined use of Doppler sonography and $\mathrm{MRI}^{37}$;

- angiography to demonstrate the main blood vessels supplying sacrococcygeal teratomas - the middle and lateral sacral and gluteal branches of the internal iliac artery and a branch from the profundus femoris artery; as hypervascularity, irregularly encased vessels, arteriovenous shunting, and serpentine dilated veins may be seen in malignant as well as in benign tumours, angiography is not conclusive in distinguishing benign from malignant disease $^{8}$;

- Gross et al have discussed the role of myelography in evaluating the intraspinal extension of sacrococcygeal teratomas. ${ }^{38}$

\section{Management at delivery}

The management of sacrococcygeal teratomas depends on fetal gestational age and wellbeing, the presence of associated anomalies, and tumour vascularity assessed by colour flow Doppler. ${ }^{18}$ Highly vascular tumours should be delivered by caesarean section to avoid risk of haemorrhage during the second stage of labour. ${ }^{18}$ Robertson et al reported devascularisation of a sacrococcygeal teratoma to decrease the chance of spontaneous haemorrhage in a preterm neonate ${ }^{39}$; antenatal ultrasound had confirmed the highly vascular nature of tumour, with several large feeding vessels arising from the internal iliac arteries. Both internal iliac arteries and the small middle sacral artery were ligated, after stabilisation of respiratory status. $^{39}$

Delivery should be conducted in a tertiary hospital. The size of a sacrococcygeal teratoma determines the mode of delivery. Non-vascular tumours and those less than $10 \mathrm{~cm}$ in diameter may be delivered vaginally. ${ }^{18}$ However, Gross et al recommended caesarean delivery in all fetuses with sacrococcygeal teratomas of more than $5 \mathrm{~cm}$ diameter, to minimise the risk of rupture and haemorrhage..$^{40}$ Tumour size has been more than $10 \mathrm{~cm}$ in almost all reported cases with difficult deliveries. ${ }^{14}{ }^{41-43}$ Teratomas should be well protected after delivery because erosion of the surface may precipitate bleeding before surgical excision is undertaken. ${ }^{44}$ Blood loss in large and even benign tumours has been reported to be substantial, sometimes equalling the patient's blood volume. ${ }^{46}$ Most reviews report mortality ranging from around $5 \%$ to $9 \%$ following exsanguinating haemorrhage. ${ }^{14} 34748$

Grosfeld et al reported disseminated intravascular coagulation in a neonate who suffered tumour rupture and haemorrhage during delivery. ${ }^{34}$ The aetiology of such clotting abnormalities is probably complex and multifactorial. ${ }^{45}$ Extensive disruption of large, defective tumour endothelium during labour and delivery could precipitate fulminant disseminated intravascular coagulation. Trauma to the sacrococcygeal teratoma during delivery may cause entry of tissue thromboplastin into the blood stream, resulting in activation of the coagulation cascade. ${ }^{45}$ Good vascular access and adequate supply of blood products is thus necessary before surgery.

During surgery, precautions must be taken to prevent hypothermia, which is easily precipitated because of the large surface area and the vascularity of the tumour. ${ }^{44}$ The treatment of choice for sacrococcygeal teratoma is early surgical resection with complete excision of the coccyx, because microscopic nests of neoplastic cells are commonly found in or immediately adjacent to the coccyx. ${ }^{5}{ }^{15} \mathrm{~A}$ recurrence rate of $37 \%$ was reported when the coccyx was not removed completely. ${ }^{51}$ Patients with malignant sacrococcygeal teratomas are managed after surgery with irradiation if residual disease is present, and always with combination chemotherapy. ${ }^{115}$

\section{Postoperative complications}

The main postoperative complication is wound infection because of the proximity to the anus of the surgical site and the skin flaps that may be needed. ${ }^{89}$ Bladder dysfunction may occur. ${ }^{8447}$ 
Follow up/recurrence

The recurrence rate of sacrococcygeal teratomas varies between $7.5 \%$ and $22 \%{ }^{48}{ }^{49}$ In contrast to previous reports, ${ }^{14}{ }^{50}$, Bilik et al found a higher recurrence rate for primary sacrococcygeal teratomas with larger mean diameters (11.1 (3.2) $\mathrm{cm}$ with recurrence $v 7.9(4.4) \mathrm{cm}$ with no recurrence (mean (SD)); $\mathrm{p}=0.07) .{ }^{49}$ They concluded that despite multiple histological sections in large primary tumours reported as benign, these large tumours may well harbour undetectable small foci of malignant endodermal sinus cells.

Serum $\alpha$ fetoprotein in the first month of life and immunohistochemical markers are not reported to be of prognostic significance. ${ }^{50}$ However, after total resection of primary sacrococcygeal teratoma together with the coccyx, a raised serum $\alpha$ fetoprotein has been found to be a reliable marker of recurrence of poorly differentiated yolk sac tumours. ${ }^{51}$ Bilik et al reported markedly raised serum $\alpha$ fetoprotein in malignant recurrent compared with benign sacrococcygeal teratomas. ${ }^{49}$ As none of the variables except tumour size was found to be a reliable predictor of recurrence, these investigators concluded that meticulous routine physical examination is essential every three to six months for at least the first three postoperative years to detect recurrence. ${ }^{49}$ They also emphasised the importance of regular serum $\alpha$ fetoprotein measurements, prompt radiographic investigations to detect possible recurrences (for example where there is raised $\alpha$ fetoprotein but clinically undetectable tumour), and the need to regard every recurrence as potentially malignant. ${ }^{49}$

The potential for late occurrence of malignancy in mature sacrococcygeal teratoma is well documented..$^{52}$ Lack et al reported infiltration of virtually the entire coccyx with an adenocarcinoma 40 years after the resection at two months of age of a congenital mature teratoma. ${ }^{52}$ Lahdenne et al followed up 45 patients (aged 4-43 years, mean 21.5 years) for detection of late recurrences. ${ }^{53}$ All had been operated on in infancy for benign sacrococcygeal teratomas. Three recurrences (two benign and one malignant) were diagnosed 21 to 43 years after the initial diagnosis and operative treatment; coccygectomy had not been performed as a primary procedure in patients with malignant recurrence. Rescorla et al reported recurrent disease in nine of 80 patients $(11 \%)$ with mature teratomas, at between six and 34 months after resection. ${ }^{9}$ Three had metastatic disease. Two were long term survivors after surgery alone. Six were alive at a mean follow up time of 114 months after chemotherapy (5) and pelvic irradiation (1). One patient with metastatic disease was lost to follow up. This series also included cases of immature teratoma and endodermal sinus tumour (EST). Of 24 children with immature teratoma followed up for an average of 39 months, one $(4.2 \%)$ had a rising $\alpha$ fetoprotein level 6 months after the initial procedure and required resection. The child was free of disease 33 months after the second procedure. Of the 13 neonates and infants with EST, nine under- went early surgery with complete resection. Seven were long term survivors. Two died from non-disease-related causes. One child whose lesion was observed after birth presented with metastatic disease at 10 months of age and underwent incomplete resection at that time. She died despite adjuvant treatment. Three other patients presenting between seven and 30 months of age without evidence of metastatic disease underwent complete resection and were long term survivors. ${ }^{9}$

Schropp et al reported their experience with sacrococcygeal teratomas in children over four decades (1950 to 1990). ${ }^{54}$ Of 73 teratomas, 57 $(78 \%)$ were benign and $16(22 \%)$ malignant. There were five recurrences in children with benign disease, only one of which did not have an initial coccygectomy. The average age at presentation of benign sacrococcygeal teratomas was 20 days $v 468$ days in those with malignant disease. Seven of the 16 children with malignant sacrococcygeal teratomas were long term survivors. The average survival was 9.3 months in the nine children who died (range 1 to 28 months). Only two of 10 cases (diagnosed between 1976 and 1991) undergoing chemotherapy and radiotherapy for malignant disease died with disease ( $80 \%$ survival). Three deaths occurred in those with benign sacrococcygeal teratomsa ( $95 \%$ survival). One neonate died in the immediate postoperative period because of complications (rupture of hepatic subcapsular haematoma and disseminated intravascular coagulation with sepsis). ${ }^{54}$ Maturation from malignant to benign sacrococcygeal teratoma after chemotherapy (pseudoretroconversion) has been reported. ${ }^{54} 55$

\section{Sequelae of sacrococcygeal teratoma}

The type of the teratoma and the surgical approach both contribute to functional sequelae. Tumours with a large presacral component may present with lower extremity weakness or paralysis, constipation, abdominal distension, and urinary tract symptoms secondary to bladder outlet obstruction. ${ }^{56-58}$ Isolated cases of lower extremity weakness or paralysis and bladder dysfunction have been described postoperatively, particularly in association with malignant lesions. Many of the previously reported large series did not report long term faecal and urinary incontinence. ${ }^{34} 475659-63 \mathrm{En}$ gelskirchen et al reported electromanometrically detected rectal and bladder dysfunction in $40 \%$ of children following excision of sacrococcygeal teratoma, although none had overt incontinence. ${ }^{63}$ Malone et al reported on 27 patients followed up for a mean period of five years (range $2-12) .{ }^{64}$ Eleven $(41 \%)$ had some form of functional impairment. Faecal and urinary incontinence were present in nine. Two had lower extremity weakness because of sciatic nerve palsy. ${ }^{64}$ Tumours with large intrapelvic extensions requiring an abdominoperitoneal approach for resection were associated with a higher incidence $(67 \%)$ of functional sequelae. It was concluded that it was impossible to determine retrospectively whether dysfunction was caused by the tumour 
itself or by the surgery. ${ }^{64}$ Rintala et al evaluated quality of life and faecal continence in 26 adult patients (mean age 30 years) who had undergone surgery for benign sacrococcygeal teratoma in infancy. ${ }^{65}$ Good faecal continence was reported by $88 \%$ of these patients. However, only $27 \%$ had completely normal bowel habits. Some faecal soiling was present in $27 \%$. No correlation between the severity of anorectal malfunction and the degree of intrapelvic extension of the tumour was found. Other health problems, including urinary incontinence, were reported by 13 patients $(50 \%)$. All control subjects ( 26 healthy patients of similar age and sex distribution) had good faecal continence; $77 \%$ had completely normal bowel habits. ${ }^{65}$ Hypergonadotropic hypogonadism and sperm abnormalities have been reported in men born with benign sacrococcygeal teratomas. ${ }^{66}$ Such patients may have Leydig cell dysfunction, abnormal spermatogenesis, or both. The association of these with sacrococcygeal teratomas may reflect a congenital germ cell defect. ${ }^{66}$

1 Arceci RJ, Weinstein HJ. Neoplasia. In: Avery GB, Fletcher MA, MacDonald MG, eds. Neonatology, pathophysiology and management of the newborn. Philadelphia: J B Lippincott, 1994:1219-20.

2 Winderl LM, Silverman RK. Prenatal identification of a completely cystic internal sacrococcygeal teratoma (type IV). Ultrasound Obstet Gynecol 1997;9:425-8.

3 Ballantyne JW. Teratologie. Williams and Nougate, 1874

4 Gross RE, Clatworthy HW, Meeker IA. Sacrococcygeal teratoma in infants and children: report of 40 cases. Surg Gynecol Obstet 1951;92:341-52.

5 Izant RJ, Filston HC. Sacrococcygeal teratomas: analysis of 43 cases .Am F Surg 1975;130:617-21.

6 Ein SH, Adeyemi SD, Mancer K. Benign sacrococcygeal teratoma in infants and children: a 25 year review. Ann Surg teratoma in infants

7 Moazam F, Talbert JL. Congenital anorectal malformations: harbingers of sacrococcygeal teratomas. Arch Surg 1985; 120:856-9.

8 Keslar PJ, Buck JL, Suarez ES. Germ cell tumors of the sacrococcygeal region: radiologic-pathologic correlation. Radiographics 1994;14:607-22.

9 Rescorla FJ, Robert SS, Arnold GC, et al. Long-term outcome for infants and children with sacrococcygeal teratoma: a report from the Children's Cancer Group. $\mathcal{F}$ Pediatr Surg 1998;33:171-6.

10 Juric-Lekic G, Trosic M, Svajger A. Lentinoids within sacrococcygeal teratoma: origin by transdifferentiation? Hum Pathol 1993;24:227-9.

11 Consolato S, Volker E, Bernhard B. Huge fetal sacrococcygeal teratoma with a completely formed eye and intratu-
moral DNA ploidy heterogeneity. Pediatr Dev Pathol 1999;2: moral

12 Valdisserri RO, Yunis EJ. Sacrococcygeal teratomas. Cancer 1981;48:217-21.

13 Parizek J, Nemecek S, Pospisilova B, et al. Mature sacrococcygeal teratoma containing the lower half of a human body. Child Nerv Syst 1992;8:108-10

14 Altman RP, Randolph JG, Lilly JR. Sacrococcygeal teratoma. American Academy of Pediatrics Surgical Section survey.1973. F Pediatr Surg 1974;9:389-98.

15 Brinker MR, Sheldin RG, Moynihan PC. Sacrococcygea teratoma in children $\mathcal{F}$ La State Med Soc 1989;141:26-31.

16 Murphy JJ, Blair GK, Fraser GC. Coagulopathy associated with large sacrococcygeal teratomas. F Pediatr Surg 1992;27: 1308-10.

17 Graf JL, Housely HT, Albanese CT, et al. A surprising histological evolution of preterm sacrococcygeal teratoma. $\mathcal{F}$ logical evolution of preterm
Pediatr Surg 1998;33:177-9.

18 Kum CK, Wong YC, Prabhakaran K. Management of fetal sacrococcygeal teratoma. Ann Acad Med 1993;22:377-80.

19 Holterman AX, Filiatrault D, Lallier M, et al. The natural history of sacrococcygeal teratomas diagnosed through routine obstetric sonogram: a single institution experience. $\mathcal{F}$ Pediatr Surg 1998;33:899-903.

20 Carney JA, Thompson DP, Johnson CL. Teratomas in children: clinical and pathologic aspects. $f$ Pediatr Surg 1972;7:271-86

21 Subbarao P, Bhatnagar V, Mitra DK. The association of sacrococcygeal teratoma with high anorectal and genital malformations. Aust NZ F Surg 1994;64:214-15.

22 Vogl D, Riel KA. Prasakraler Tumor mit sakraler Meningozele. $Z$ Kinderchir 1988;43:361-4.

23 Lahdenne P, Heikinheimo M, Jaaskelainen J, et al. Vertebral abnormalities associated with congenital sacrococcygeal teratomas. F Pediatr Orthop 1991;11:603-7.
4 Gergely RZ, Eden R, Schinfrin BS. Antenatal diagnosis of congenital sacral teratoma. $\mathcal{F}$ Reprod Med 1975;24:229-31.

25 Kohga S, Nambu T, Tanaka K. Hypertrophy of placenta and sacrococcygeal teratoma. Virchows Arch A 1980;386: 223-9.

26 Bond SJ, Harrison MR, Schmidt KG. Death due to high-output cardiac failure in fetal sacrococcygeal teratoma. 7 Pediatr Surg 1990;25:1287-91.

27 Inou M, Kubota A, Hasegawa T, et al. Antenatal diagnosis of sacrococcygeal teratoma with hydrops fetalis. Eur $\mathcal{F}$ Pediatr Surg 1994;4:125-7.

28 Zaninovic AC, Westra SJ, Hall TR, et al. Congenital bladder rupture and urine ascites secondary to a sacrococcygeal teratoma. Pediatr Radiol.1992;22:509-11.

29 Elchalal U, Ben-Shachar I, Nadjari M, et al. Prenatal diagnosis of acute bladder distension associated with fetal sacrococcygeal teratomaa case report. Prenat Diagn 1995; 15:1160-4

30 Garcia AM, Morgan WM, Bruner JP. In utero decompression of a cystic grade IV sacrococcygeal teratoma. Fetal Diagn Ther 1998;13:305-8.

31 Chisholm CA, Heider AL, Kuller JA, et al. Prenatal diagnosis and perinatal management of fetal sacrococcygeal teratoma. Am $\mathcal{F}$ Perinatol 1999;16:47-50.

32 Nakayama DK, Killian A, Hill LM, et al. The newborn with hydrops and sacrococcygeal teratoma. $\mathcal{f}$ Pediatr Surg 1991;26:1435-8.

33 Mahour GH, Wooley MM, Trivedi SN. Sacrococcygeal teratoma: a 33 year experience. $\mathcal{F}$ Pediatr Surg 1975;10:183-

34 Grosfeld JL, Ballantine TVN, Lowe D. Benign and malignant teratomas in children: analysis of 85 patients. Surgery 1976;80:297-305.

35 Feldman M, Byrne P, Johnson MA, et al. Neonatal sacrococcygeal teratoma: multiimaging modality assessment. Am F Obstet Gynecol 1990;163:675-82.

36 Cohen MD. Clinical utility of magnetic resonance imaging in pediatrics. Am 7 Dis Child 1986;140:947-56.

37 Hata K, Hata T, Kitao M. Antenatal diagnosis of sacrococcygeal teratoma facilitated by combined use of Doppler sonography and MR imaging. Am 7 Roentgenol 1991;156: 1115-16.

38 Gross RE, Clatworthy HW, Meeker IA. Sacrococcygeal teratoma in infants and children: report of 40 cases. Surg Gynecol Obstet 1951;92:309-18.

39 Robertson FM, Crombleholme TM, Frantz ID. Devascularization and staged resection of giant sacrococcygeal teratoma in the preterm infant. F Pediatr Surg 1995;30:30911.

40 Gross SJ, Benzie RJ, Sermer M, et al. Sacrococcygeal teratoma: prenatal diagnosis and management. Am $\mathcal{F}$ Obstet Gynecol 1987;156:393-6.

41 Viller MR. Dystocia caused by sacrococcygeal teratoma. Two case reports. Obstet Gynecol 1969;34:783.

42 Weiss DB, Wajntraub G, Abulafla Y. Vaginal surgical intervention for sacrococcygeal teratoma obstructing labour. Acta Obstet Gynecol Scand 1976;55:183-6.

43 Schiffer MA, Greenberg E. Sacrococcygeal teratoma in labour and the newborn. Am f Obstet Gynecol 1956;72: 1054-8.

44 Teitelbaum D, Teich S, Cassidy S, et al. Highly vascularized sacrococcygeal teratoma: description of this atypical variant and its operative management. F Pediatr Surg 1994;29:98101.

45 Murphy JJ, Blair GK, Fraser GC. Coagulopathy associated with large sacrococcygeal teratoma. F Pediatr Surg 1992;27: 1308-10.

46 Smith B, Passaro E, Clatworthy HW. The vascular anatomy of sacrococcygeal teratoma; its significance in surgical management. Surgery 1961;49:534-9.

47 Noseworthy J, Jack EE, Kosakewich HPW. Sacrococcygeal germ cell tumors in childhood: an updated experience with 118 patients. F Pediatr Surg 1981;16:358-64.

48 Dewan PA, Davidson PM, Campbell PE. Sacrococcygeal teratoma: has chemotherapy improved survival? $\mathcal{F}$ Pediatr Surg 1987;22:274-7.

49 Bilik R, Shandling B, Pope M, et al. Malignant benign neonatal sacrococcygeal teratoma. F Pediatr Surg 1993;28:115860.

50 Lahdenne P, Heikinheimo M, Periko M. Cell differentiation in a sacrococcygeal teratoma. An immunohistochemical and follow up study. Pathol Res Pract 1990;186:336-43.

51 Crussi FG, Winkler RF, Mirkin DL. Sacrococcygeal teratoma in infant and children. Arch Pathol Lab Med 1978; 102:420-5.

52 Lack EE, Glaun RS, Hefter LG, et al. Late occurrence of malignancy following resection of a histologically mature sacrococcygeal teratoma. Arch Pathol Lab Med 1993;117: $724-7$

53 Lahdenne P, Heikinheimo M, Nikkanen V, et al. Neonatal benign sacrococcygeal teratoma may recur in adulthood and give rise to malignancy. Cancer 1993;15:3727-31.

54 Schropp KP, Lobe TE, Rao B. Sacrococcygeal teratoma: the experience of four decades. F Pediatr Surg 1992;27:1075-9.

55 Cranston PE, Smith EE, Hamrick-Turner J Emergence of mature teratoma following treatment of sacrococcygeal endodermal sinus tumour: CT and MR imaging with pathol correlation. Pediatr Radiol 1994:24:239-40.

56 Leung AKC, Rubin SZ, Seagram GF, et al. Sacrococcygeal teratoma. Aust Paediatr f 1985;21:123-5.

57 Whalen TV, Mahour GH, Landing BH, et al. Sacrococcygeal teratomas in infants and children. Am $\mathcal{F}$ Surg 1985;150: geal terato 373 . 
58 Ashcraft KW, Holder TM. Hereditary presacral teratoma. $\mathcal{F}$ Pediatr Surg 1974;9:691-7.

59 Tapper D, Lack EE. Teratomas in infancy and childhood. A 54 year experience at the Children's Hospital Medical Cen54 year experience at the Children's
tre. Ann Surg 1983;198:398-410.

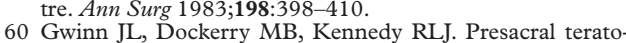
mas in infancy and childhood. Pediatrics 1955;16:239-49.

61 Billmire DF, Grosfeld JL. Teratomas in childhood: an analysis of 142 cases. F Pediatr Surg 1986;21:548-51.

62 Kirk D, Lister J. Urinary complications of sacrococcygeal teratoma. Z Kinderchir 1976;18:294-304.
63 Engelskirchen R, Holschneider AM, Rhein R, et al. Sacrococcygeal teratomas in children. An analysis of long term results in 87 children. Z Kinderchir 1987;42:358-61.

64 Malone PS, Spitz L, Kiely EM, et al. The functional sequela of sacrococcygeal teratoma f Pediatr Surg 1990;25:679-80.

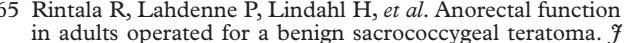
in adults operated for a benign

66 Lahdenne P, Dunkel L, Heikinheimo M, et al. Hypergonadotropic hypogonadism and sperm abnormalities with benign sacrococcygeal teratomas. F Androl 1991;12:226-30. 